\title{
Comparative Analysis of Coverall Prototype of Several Countries \\ in Virtual Fitting
}

\author{
Xin Zhang ${ }^{1, a}$, Ming-Hai Cui ${ }^{1, b}$ \\ 1 Beijing Institute of Fashion Technology, China \\ aBIFTzhangx@163.com , 'bfzycmh@bift.edu.cn
}

Keywords: Virtual fitting, Coverall prototype, Comparison of similarities and differences.

Abstract: The coverall prototype of several countries were compared and analyzed with the virtual fitting software Marvelous Designer 5 produced by South Korean CLD. Control variables were unified to paint paper patterns. The differences and similarities of Germany Muller prototype, France prototype, Japan's Torso prototype, Italian simple prototype, and British coverall prototype were compared in the aspect of the principles and methods of plane drawing, profile comparison, setting of dart and cutting line, which provided reference for dress designing and producing.

\section{Introduction}

Prototype refers to the basic form or pattern before the application of various practical changes ${ }^{[1]}$. As for costume modeling, prototype refers to the basic paper pattern used in flat pattern tailoring, that is, simple and three-dimensional paper pattern of clothing without any changing style ${ }^{[1]}$. Domestic research methods are not mature, and coverall prototype is still in the theoretical stage. There have been a lot of studies of prototype, but there is no study of coverall prototype. In China, the earliest prototype is the prototype of the upper body launched by Donghua University in 1988 according to the study on the Chinese body figure and clothing culture.

Foreign prototype has involved coverall prototype, that is, setting the measuring part of human body for measurement. The law of the changes of the size of body's each place was summarized according to the statistical analysis of the measured data. On the basis of the law of the measured data, new method of pattern making was studied. Many foreign countries have body prototypes suitable for the body figure of their people. European and American countries such as Germany, France, Italy, and Britain designed the prototype structure by measuring the size of the body's part. The form of expression of prototypes is different according to body figures. The mainstream prototypes of foreign clothing education were compared and analyzed in the aspect of principles and methods of drawing, profile comparison, setting of dart and cutting line, and aesthetics of prototype, which provided theoretical foundation for clothing designing and producing.

\section{Comparison of the Plane Drawing and Method of the Prototypes of Several Countries}

Through reviewing literature ${ }^{[2,3,4]}, 5$ commonly used paper patterns of prototype of female coats in five countries with developed clothing industry, namely, Germany Muller prototype, France prototype, Japan's Torso prototype, Italian simple prototype and British coverall prototype. The control variables of the five prototypes were unified to paint paper pattern in garment $\mathrm{CAD}$, that is, chest circumference was $84 \mathrm{~cm}$; waistline was $68 \mathrm{~cm}$; hipline was $90 \mathrm{~cm}$, and the female coverall prototype of these countries were compared. Paper patterns are shown in Figure 1-5. 


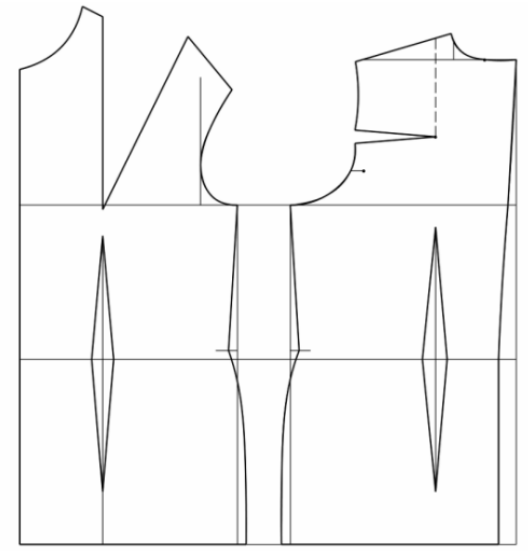

Fig.1 Germany Muller prototype

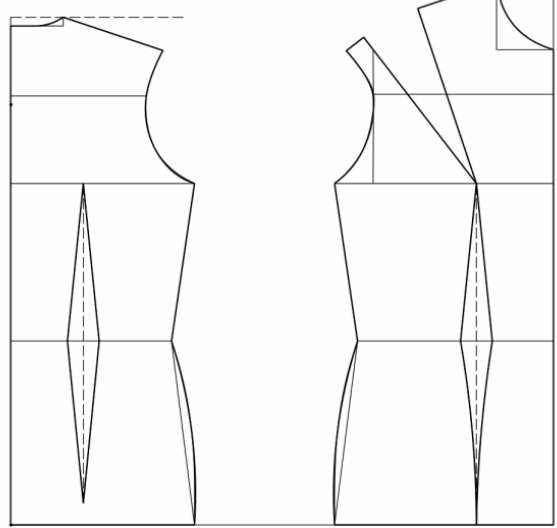

Fig.2 France prototype

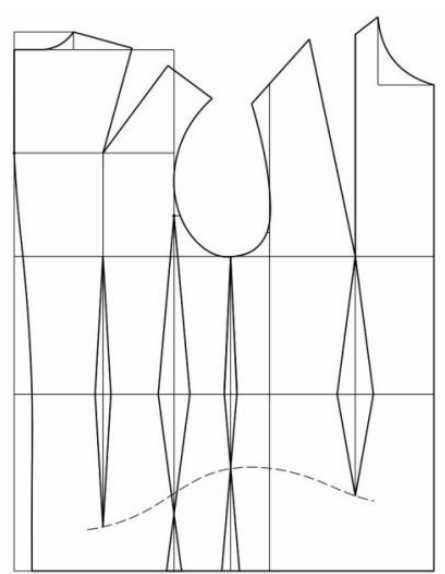

Fig.3 Japan's Torso prototype

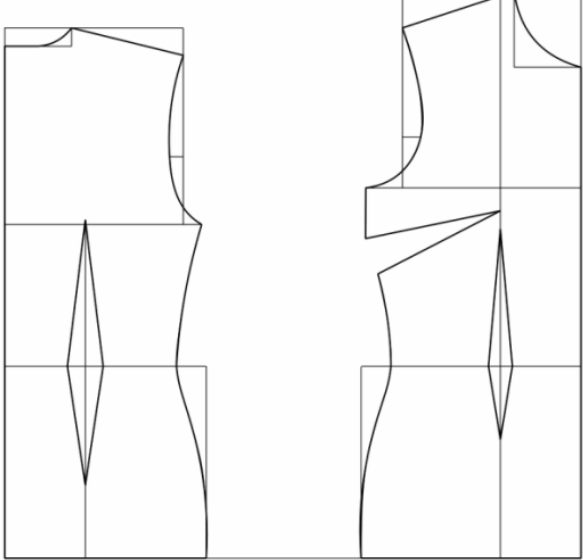

Fig.4 Italian simple prototype

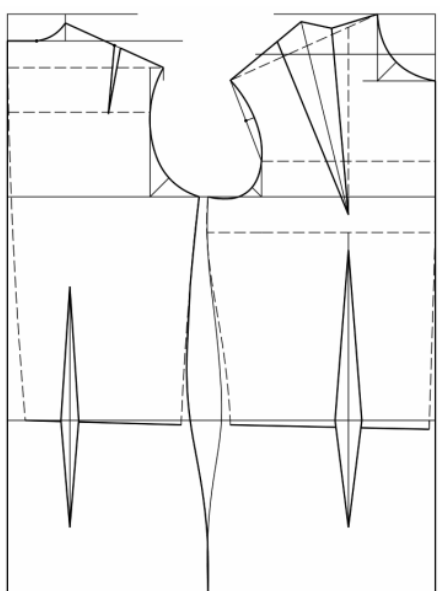

Fig.5 British coverall prototype

There are a variety of methods to draw the prototypes. A number of foreign and domestic practices proved the scientificity and effectivenss of structural design methods. The most basic condition of prototype is simple structure. In addition, prototype can be painted with the size convenient for measurement. Different prototypes have different principles and methods of drawing. Because there are differences in the standard size of human body in different countries, there are more structural design methods.

Germany Muller prototype, France prototype, Italian simple prototype e and British coverall prototype adopt the method with more measuring and confirming the size. All countries have their own prototypes, and different specific clothing paper patterns are made according to the different sizes of human body. The difference of prototype depends on the variation of the standard size of human body, confirmed size and calculating formula and method.

Based on the expansion graph of the paper model of human body, Japan Torso prototype is the sample plate obtained by setting the base line and observing the features of the opening and crossing size determined by the relationship of the size of each part and the concave and convex parts of human body ${ }^{[3]}$. The Torso prototype can be made in any size.

\section{Comparative Analysis of Virtual Fitting}

The prototype of several countries was compared and analyzed with the virtual fitting software Marvelous Designer 5 developed by South Korean CLD company. Marvelous Designer can make various clothings with different materials. The drapability of textile can be simulated by the 
software. According to previous studies and literature[5], the regression equation of drape coefficient of the simulated textile was obtained. Drape coefficient had a linear relationship with Stretch, Shear, Bending and Density, and the fabric attribute parameters including Bending and Density had the most significant effect on drape coefficient. The drape coefficient of the simulated textile was highly correlated to Bending, moderately correlated to Density, weakly correlated to Stretch and Shear, and had no relation with Buckling Ratio and Buckling Stiffness. Therefore, when the fitting experiment is carried out, the value of D_Cotton_CLO_v2 can be referred. The value of Bending was 55; Density, 35; Stretch, 32; Shear, 23; Buckling Ratio, 50; Buckling Stiffness, 30; shape coefficient, $65.7 \%$; the fabric attribute of grey cloth was restored to the largest extend to realize the best effect. Figure 6 is the detail table of the classification of fabric attributes and physical attributes in Marvelous Designer 5:

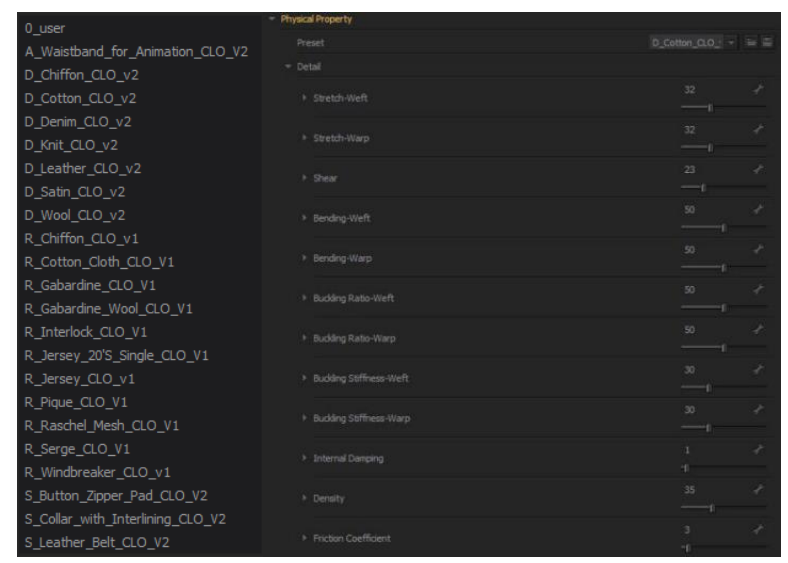

Fig.6 Classification of fabric attributes and and physical attributes in Marvelous Designer 5

Fitting model was the160/84A standard naked female mannequin produced by Japan KIIYA. Meanwhile, the "dxf" format files of the prototype paper patterns of five styles of female coat made by clothing CAD software were introduced to Marvelous Designer 5 for virtual fitting and effect evaluation to observe the degree of comfort under the situation of prototype dress. The virtual fitting software showed the distribution and size of pressure through the change of the force color of the contact surface and distribution of pressure spot. Pressure distribution can show the force degree of prototypes, that is, the degree of comfort of dressing. The specific fitting effects are shown in Figure 7-11:

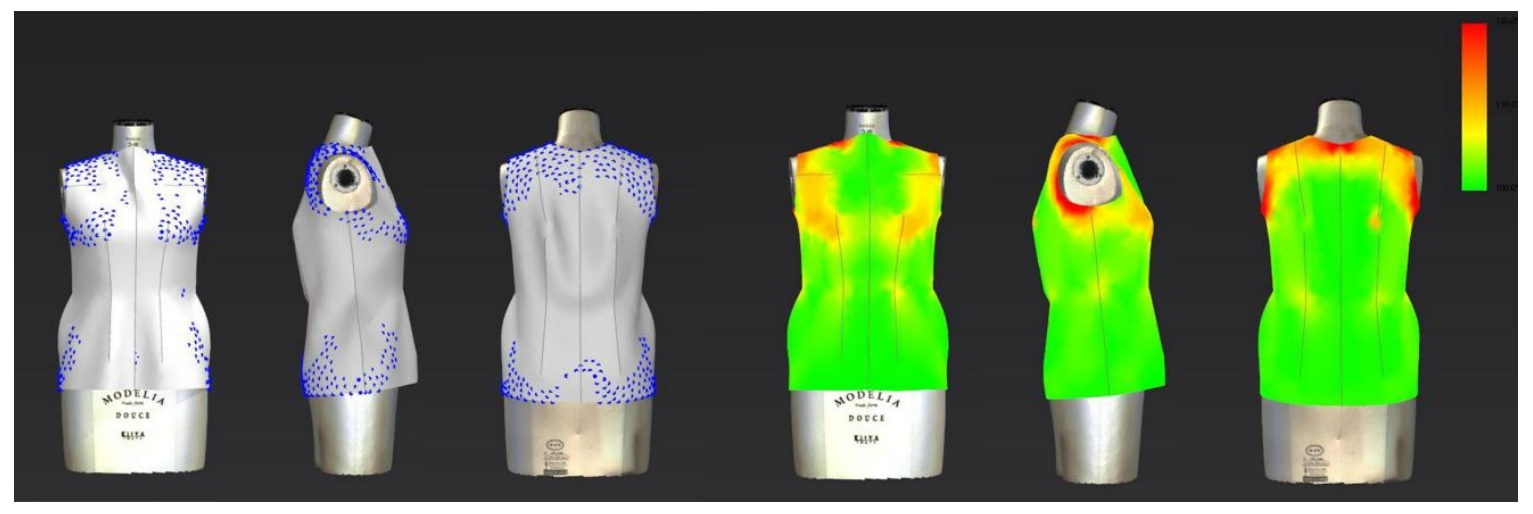

Fig.7 Pressure distribution of Germany Muller prototype 


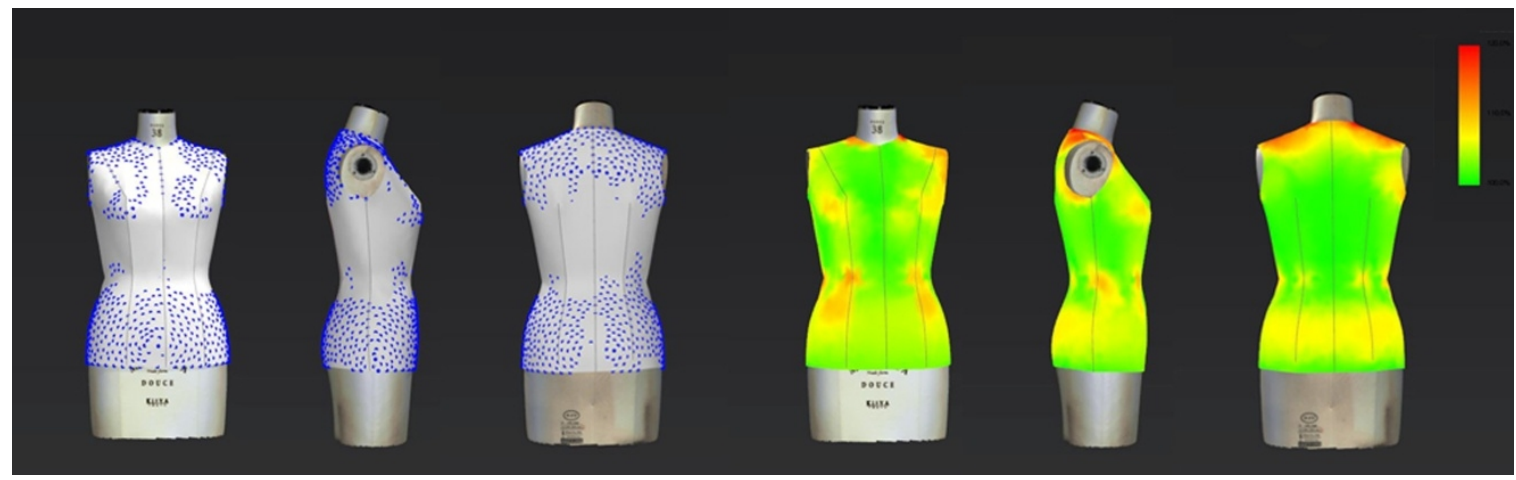

Fig.8 Pressure distribution of France prototype

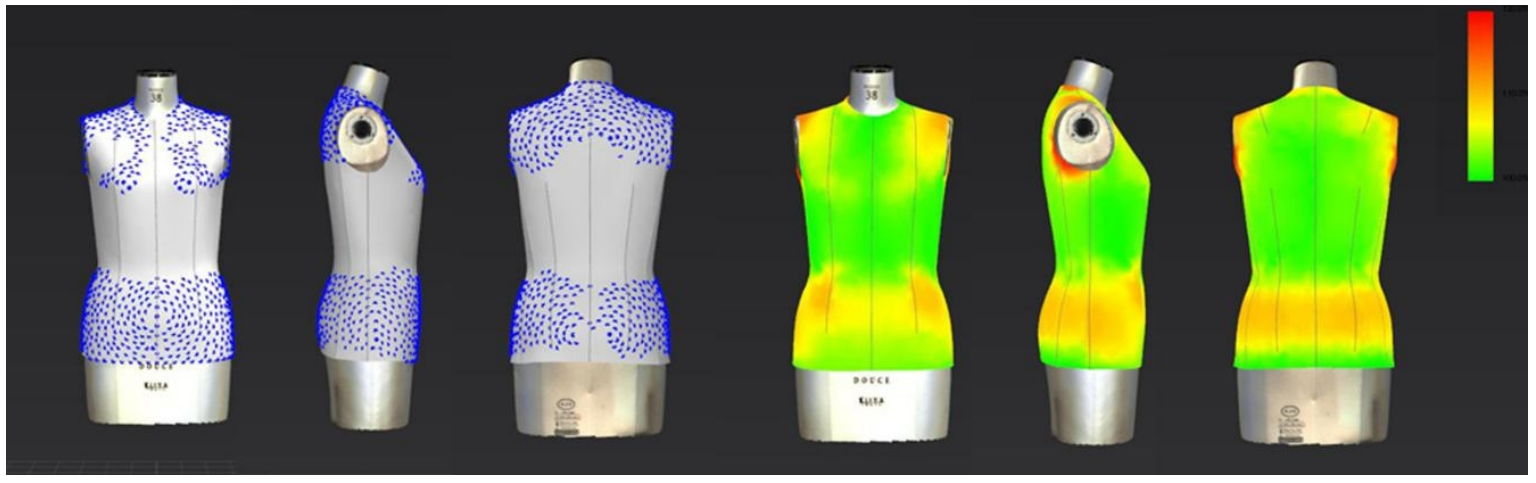

Fig.9 Pressure distribution of Japan's Torso prototype

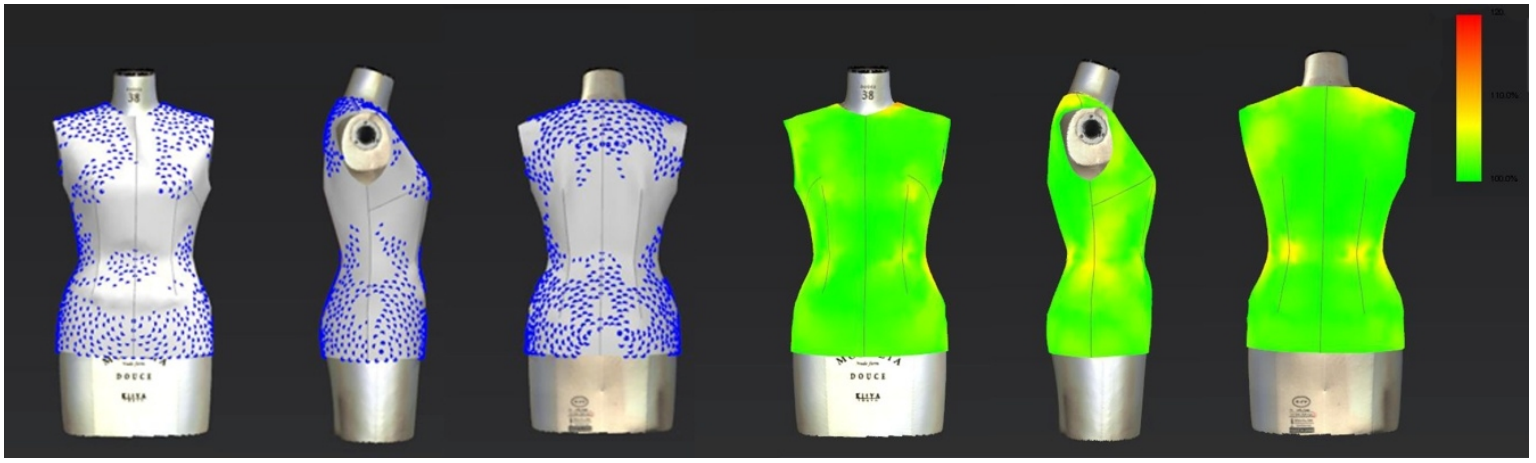

Fig.10 Pressure distribution of Italian simple prototype

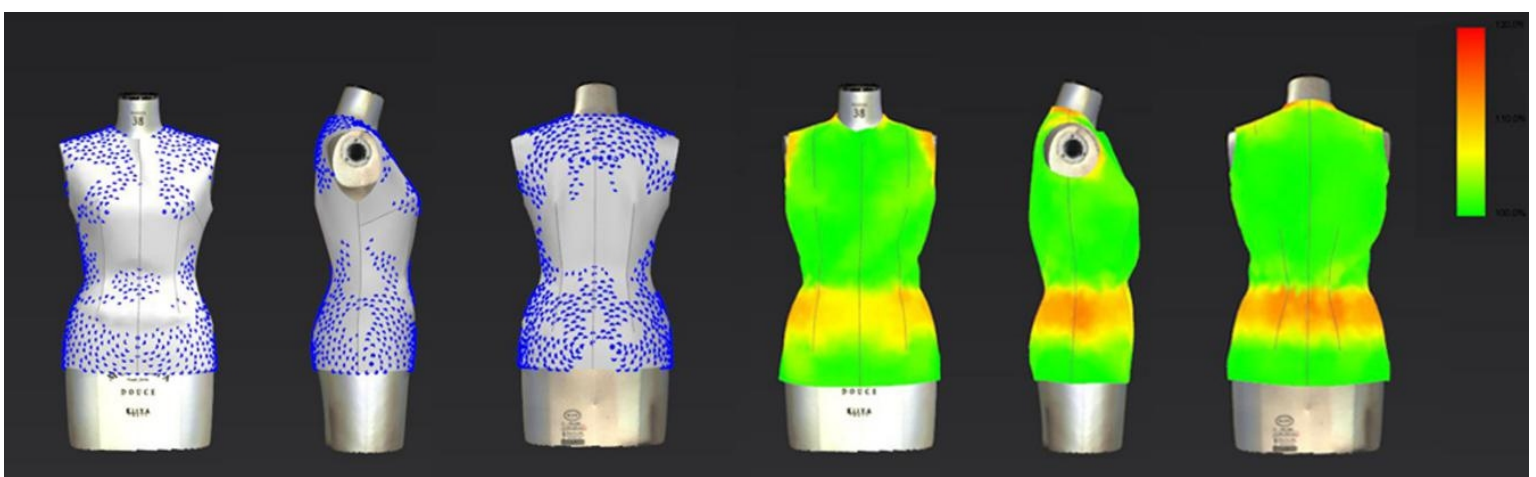

Fig.11 Pressure distribution of British coverall prototype

\section{Comparative Analysis of Profile}

Profile can reflect clothing style and body shape. Profile depends on the degree of fitness of garments. Clothing profile can be divided into five categories, namely $\mathrm{H}, \mathrm{A}, \mathrm{T}, \mathrm{X}$, and $\mathrm{O}$. The prototypes in literature were all coverall prototypes, and four of them were close-fitting coverall prototypes which were X, namely, France prototype, Japan Torso prototype, Italian simple 
prototype, and British coverall prototype. The three-dimensional constitution form of Germany Muller prototype was H. It can be seen from the pressure spot distribution that the degree of fitness of the four close-fitting coverall prototypes was higher than Germany Muller prototype.

\section{Analysis of the Design of Dart and Cutting Line}

Many parts and structures of clothing are expressed in the form of dart ${ }^{[6]}$. The dart of front close is the most used and has the most changes. According to the location of dart, it can be classified into the shoulder dart, collar dart, armhole dart, waist dart, underarm dart and front and middle dart. According to the location of dart, collar dart, armhole dart, underarm dart and front and middle dart are called breast dart. The size and number of dart have a direct effect on the degree of fitting of prototypes. There are various patterns of cutting line which plays an important role in the degree of fitness and aesthetics and can be classified into decorative cutting line and functional cutting line. As an important cutting line in prototype design, princess line is essentially to connect dart to line. Dart value is formed by the curve and residual quantity between chest and waist ${ }^{[7]}$. The setting of cutting line can show the outside contour of body to the largest extent and form a reasonable curved surface shape.

It can be seen from Figure 7-11 and paper pattern drawing:

Japan's Torso prototype had the most darts, and Italian simple prototype had the largest dart, and its effect of virtual fitting was better than other prototypes. In the five prototype paper patterns, the darted position of the breast dart and three prototypes was in the shoulder, and one was in the side seam, and one was in the armhole. The dart point of the breast dart of France prototypes and Japan's Torso prototypes was on the bust line. The dart point of the breast dart of Italian simple prototype and British coverall prototype was below the bust line.

(1) The front and middle position of Germany Muller prototype deviated to side seam by $2 \mathrm{~cm}$, and the front neckline depth was small, leading to high front central line and extra fold in the virtual fitting by Marvelous Designer 5. Meanwhile, the side seam line had break angle and did not change with body curve. The hollow size of the front armhole was small and the bottom point of armhole was above the bust line. The armhole line was too small and did not accord with the relationship between clothe body and armhole as well as the basic form of human body motion. The dart value of the back shoulder dart was large, and there was protuberance on the dart point of shoulder dart and waist dart. The garment ease of the waist part was small, so the whole prototype was loose and unfitted on the 160/84A standard mannequin.

(2) The degree of fitness of France prototype was higher. It transferred and eliminated the clothe body float remainder in the cutting way of princess line. The back part set waist dart and the dart value of front and back part was larger. Viewing the fitting effect of virtual fitting with Marvelous Designer 5 from side, France prototype had an empty shoulder tip, higher shoulder line, and shallow front armhole. However, viewing the form of pressure points and color distribution of pressure surface as a whole, the degree of fitness of France prototyoe was higher and better showed the beauty of human body curve.

(3) The ease allowance of the chest circumference of Torso prototype was smaller, so the float remainder of shoulder dart and breast dart was smaller. Because the thinnest parts of waist circumference were not in a horizontal plane, Torso prototype raised the bust line by $0.5 \mathrm{~cm}$ on the side seam. The form of Torso prototype was nearly flat, wide front and back, narrow muffs, larger front shoulder slope, small back shoulder slope. Shoulder line leaned forward, and the neckline was smaller. The ease allowance of waist circumstance of Torso prototype was larger. Auxiliary dart was made in side seam and back side dart to eliminate the float remainder of waist and realize the effect 
of fitted. Torso prototype did not involve angle. When prototypes are made, only net body size and ease allowance are necessary. The prototype not only can be accurately measureed and custom-made according to individuals, but also can satisfy the tailoring production demand by calculating with formula. It can be seen from the distribution of pressure spot and pressure surface when observing the fitting effect that the overall degree of tightness of the prototype was just right, and the position with larger curvature of mannequin showed fitness.

(4) The dart point of waist dart of Italian simple prototype was warped outward to make the prototype have protuberance on all dart points and not fit the human body curve unevenly. The hollow size of the back armhole was the smallest, and the armhole was long elliptic. Meanwhile, front shoulder had float remainder. The trend of side seam line of the prototype was too prototypical and the degree of curve was too large. There was turbulence in the side waist in virtual fitting, and the degree of fitness was not enough seen from side. The degree of fitness of the back part of Italian prototype was better, and there were obviously more pressure spots on the back part in fitting effect.

(5) The waist of British coverall prototype was loose and deviated to side seam through front central line and back central line. Meanwhile, the waist-controlled effect was realized by reducing the waist dart value. However, because the waist dart of the back part was short, and the dart value was small, there was larger float remainder in the back part. Front part adopted the cutting mode of princess line, and the part above bust line was flat. Shoulder line retracted and neckline was narrow, which made the whole neck uncomfortable in the dressing situation and the clothes body was also pulled. The position of the waist line was lower and there were many abdominal pressure spots in virtual fitting, and the abdomen was greatly stressed.

\section{Evaluation of Aesthetics}

The prototype paper patterns of five countries were all designed according to the human body shape of people of different countries. Because the standard size of human body of different countries is different, not all parts of prototype paper pattern of each country can be applied to the human body of other countries, which can be confirmed by the fitting experiment by Marvelous Designer 5 . Therefore, prototype should be designed according to specific situation.

On the basis of the comparative analysis of five commonly used prototypes, viewed from the form of distribution points and color distribution of pressure surface in virtual fitting by Marvelous Designer 5, the degree of fitness of France prototype, Japan's Torso prototype, Italian simple prototype and British coverall prototype was the highest. Excepts for Italian prototype, others adopted the cutting method of princess line to connect dart to line. The design of chest, waist and buttocks was treated to protruding chest, control waist and expand shoulders, show the beauty of body curve and meet the requirement of fitting body model. Meanwhile, the fitness degree of coverall prototype was generally higher, and expressed the body curve more finely. The model of France prototype and Japan's Torso prototype was more beautiful and more applicable, and can better satisfy the static aesthetics of human body and dynamic comfort. Later prototype design can refer to the prototype of all countries to design a Torso prototype of coat that is more suitable to the beauty of body curve.

\section{Conclusions}

Relevant theories were studied and organized and five mainstream coverall prototypes in foreign clothing education were compared and analyzed in the aspect of principles and methods of drawing, profile comparison, setting of dart and cutting line, and aesthetics of prototype. It is clear that five prototypes had their own advantages. In-depth analysis of the prototypes of these countries can 
provide reference for clothing structural design in the future.

\section{Acknowledgements}

This work was financially supported by the Beijing Key Laboratory Construction KYTG02170202.

\section{References}

[1] Miyoshi Machiko. Clothing Modeling study. Beijing: China Textile\& Apparel Press, 2006: 122.

[2] Du JS. European Fashion Pattern-Making Technology. Shanghai: Donghua University, 47-54.

[3] Ono Kiyoli. Principles of the Pattern Making of Japanese Female Ready-Made Clothes [M]. Beijing: China Youth Publishing Group, 2012,8.

[4] Natalie Bray. Pattern Designing of British Typical Clothing (Basic). Beijing: China Textile \& Apparel Press, 2001. 32-37.

[5] Wang HW, Zhang H, Guo RL. Simulation Study of Textile Drapability Based on

Three-Dimentional Clothing CAD System. Journal of Beijing Institute of Clothing

Technology(Nature Science Edition), 2015, 03: 26-32.

[6] Zhang WB. Pattern Making for Fashion Design. Beijing: China Textile\& Apparel Press, 2006: 38-121.

[7] Chen B. The Developed Design of Clothing Princess Line. Journal of Xi'an Polytechnic University, 2011. 25(3): 325-328. 